\title{
Generalized Heat Transfer between Solids and Gases under Nonlinear Boundary
}

\author{
Conditions
}

\section{AVNER FRIEDMAN*}

Communicated by D. Grubarg

Introduction. Let $B$ be a solid body with a given temperature $\psi(x)$ surrounded by a gas with temperature 1 . Then heat will transfer through the surface $\dot{B}$ of $B$ obeying the Newton Law of Cooling

$$
\frac{\partial u}{\partial \nu}=g(u)
$$

where $u$ is the temperature of the body, $\partial u / \partial \nu$ is the thermal gradient at the surface evaluated from the interior in the direction of the outward normal and $g(u)=K(1-u)$. For moderate temperatures $K$ is approximately constant; however, for very high or low temperatures $K$ will change much and nonlinearly with the temperature $u$ (for more details and references see [7]). We thus get the following boundary value problem:

$$
\begin{gathered}
\sum_{i=1}^{n} \frac{\partial^{2} u}{\partial x_{i}^{2}}-\frac{\partial u}{\partial t}=0 \text { for } x \varepsilon B, \quad t>0, \\
u(x, 0)=\psi(x) \text { for } x \varepsilon \bar{B}, \\
\frac{\partial u}{\partial \nu}=g(u) \text { for } x \varepsilon \dot{B}, \quad t>0,
\end{gathered}
$$

where $g(u)$ is nonlinear in $u$.

In what follows we shall not make use of the special form of $g$ but only make use of the following physically evident facts: $g(u)$ is continuous and decreasing in $u$ and $g(1)=0$. Furthermore, instead of considering the system (0) we shall

* Prepared under ONR Contract Nonr-222(37) (NR 041 157) with the University of California in Berkeley. 
consider the system

$$
\begin{gathered}
L u=f(x, t) \text { for } x \varepsilon B, \quad t>0, \\
u(x, 0)=\psi(x) \text { for } x \varepsilon \bar{B}, \\
\frac{\partial u}{\partial T}=g(x, t, u) \text { for } x \varepsilon \dot{B}, \quad t>0,
\end{gathered}
$$

where $L$ is a second order linear parabolic operator with coefficients depending on $x$ and $t$ (see (1.1)) and $\partial u / \partial T$ is defined in (1.4).

The system (0) for the special case $\psi(x) \equiv 0, n=1$ and $B$ a semi-infinite interval was considered by MANN \& WoLF [7] using special methods. They proved uniqueness, existence and some other properties of the solution. Their results were slightly improved by RoBerts \& MANN [15]. Very recently PADMAVALLY [9] extended the results of [7], [15] to the case where the temperature of the gas is a function $\varphi(t)$ of the time.

Our methods are completely different. They involve comparison methods with use of the maximum principle [8] and generalized simple layers which have recently been studied by Pogonzelski [12]. We proceed to describe briefly the main results of this paper.

In $\$ 1$ we prove uniqueness (Theorem 1) and boundedness (Theorem 2) of the solutions of $\left(0^{\prime}\right)$ in case $f \equiv 0$; the bound depends only on l.u.b. $|\psi(x)|$.

In $\$ 2$ we prove the existence of a solution of the system $\left(0^{\prime}\right)$ in case $f \equiv 0$. This theorem, as well as its proof, overlaps with recent results of PoGorzelski. More details are given in the remark which follows Theorem 3.

In $\$ 3$ we prove (Theorem 4) that every solution of the system $\left(0^{\prime}\right)$ with $f \equiv 0$ tends to the temperature 1 of the gas as $t \rightarrow \infty$. If the coefficients of $L$ are independent of $t$ and if $\psi(x) \equiv$ const., the solutions are monotone functions of $t$ (Theorem 5). In proving Theorem 4 we make use of the methods of $\$ 1$ and of the existence theorem of $\S 2$.

In $\$ 4$ we generalize the results of $\$ \$ 1,2$ and 3 to the following cases: (a) The temperature of the gas is a function $\varphi(t)$ of $t$. (b) The temperature of the gas varies at each point $x$ of the surface of the body and at each time $t$ in a certain way which depends only on the original temperature of the gas, and on the temperature of the body at the point $x$ and at the same time $t$. (c) Heat transfer takes place only on a part of the surface of the body, the rest of the surface is kept under a given temperature.

In $\$ 5$ we generalize most of our previous results (in particular, Theorems $1-4)$ to the case $f \neq 0$, that is, to the general non-homogeneous systems $\left(0^{\prime}\right)$.

The results of $\$ \$ 1,2$ and 3 for the special system (0) have appeared in a University of California Technical Report [5].

1. Uniqueness and Boundedness. Let $B$ be a bounded domain in the $n$ dimensional Euclidean space of variables $x=\left(x_{1}, \cdots, x_{n}\right)$ and denote by $\dot{B}$ the boundary of $B$. Let $D_{\tau}\left(D_{\infty}=D\right)$ be the topological product of $B$ with 
$0<t<\tau$ and $S_{\tau}\left(S_{\infty}=S\right)$ the topological product of $\dot{B}$ with $0<t \leqq \tau . \bar{D}_{\tau}$ and $\bar{S}_{\tau}$ will denote the closures of $D_{\tau}$ and $S_{\tau}$ respectively.

We shall assume throughout this paper that $\dot{B}$ is of class $C^{1+\delta}$ for some $0<\delta<1$. This implies that $\dot{B}$ is of class $C^{1}$ and that the angle between tangent hyperplanes to $\dot{B}$ at any two points $P$ and $Q$ is bounded by const. $|P-Q|^{\delta}$. Thus the Liapounoff conditions [12] are satisfied and the theorems of PoGonzeLskI concerning simple layers are valid. Some of our results (for instance, Theorems 1,2 ) remain true if $\dot{B}$ is only assumed to be $C^{1}$. On the other hand, there appear a few remarks in this paper where we make use of [3; Theorem 2]. We then have to assume, in addition to the above, that to every point $x$ of $\dot{B}$ there corresponds a sphere $V$ such that $V \subset B$ and $x \varepsilon \bar{V}$.

We shall consider the differential equation

$$
\begin{aligned}
L u \equiv \sum_{i, j=1}^{n} a_{i j}(x, t) \frac{\partial^{2} u}{\partial x_{i} \partial x_{i}}+\sum_{i=1}^{n} b_{i}(x, t) \frac{\partial u}{\partial x_{i}} & \\
& +c(x, t) u-\frac{\partial u}{\partial t}=0 \text { in } D \text { or } D_{\mathbf{r}}
\end{aligned}
$$

under the following conditions:

(A) $L$ is parabolic in $\bar{D}$, that is, for every point $(x, t)$ of $\bar{D}$ and for every real vector $\xi=\left(\xi_{1}, \cdots, \xi_{n}\right) \neq 0$

$$
\sum a_{i j}(x, t) \xi_{i} \xi_{i}>0 .
$$

(B) The coefficients $a_{i i}, b_{i}, c$ of $L$ are continuous in $\bar{D}$ and $c(x, t) \leqq 0$.

Together with equation (1.1) we shall consider the initial condition

$$
u(x, 0)=\psi(x) \text { on } \bar{B}
$$

and the nonlinear boundary condition

$$
\left.\frac{\partial u}{\partial T}\right|_{\left(x^{0}, t^{0}\right)}=g\left(x^{0}, t^{0}, u\left(x^{0}, t^{0}\right)\right) \quad \text { on } \quad S \quad \text { or } \quad S_{r}
$$

where

$$
\left.\frac{\partial u}{\partial T}\right|_{\left(x^{0}, t^{\circ}\right)}=\lim _{x \rightarrow x^{\circ}} \sum a_{i j}\left(x, t^{0}\right) \cos \left(\nu, x_{i}\right) \frac{\partial u\left(x, t^{0}\right)}{\partial x_{i}}
$$

and $\left(\nu, x_{j}\right)$ is the angle which the outward normal to $\dot{B}$ at $x^{0}$ forms with the $x_{j}$-axis. We shall call $\partial u / \partial T$ the transversal derivative of $u$. Using (A) we easily find that $\partial / \partial T$ is a gradient directed outward to $\dot{B}$.

The function $g(x, t, u)$ in (1.3) will be assumed to satisfy some of the following conditions:

$\left(\mathrm{G}_{1}\right) g(x, t, u)$ is continuous for $(x, t) \varepsilon \bar{S},-\infty<u<\infty$.

$\left(\mathrm{G}_{2}\right)$ For each fixed $(x, t), g$ is a (strictly) decreasing function of $u$. 
$\left(\mathrm{G}_{3}\right) g(x, t, 1) \leqq 0, g(x, t, 0)>0$.

We shall need the following lemma.

Lemma 1. Let L satisfy (A), (B) and let $w$ be continuous in $\bar{D}_{r}$ and satisfy Lw $\leqq 0$ in $D_{\tau}, w \geqq 0$ on $B$. If $w$ assumes a negative minimum in $\bar{D}_{\tau}$ then it assumes it only at points $P$ on $S_{\tau}$ and at such points $\partial w / \partial T \leqq 0$.

Proof. Let $P$ be a point where $w$ takes its negative minimum. If $P$ is not on $S_{\tau}$, then it lies in $D_{\tau}$ and by the strong maximum principle [8] it follows that $w(Q)=w(P)<0$ at all points $Q$ which can be connected to $P$ by a continuous curve whose $t$-coordinate increases monotonically. In particular, $w(Q)<0$ on $B$ which is a contradiction. We have thus proved that $P$ must lie on $S_{r}$ and that $w(Q)>w(P)$ at all points $Q$ in $D_{r}$. Noting that transversal directions are outwardly directed we conclude that $\partial w / \partial T \leqq 0$ at $P$.

Corollary. If $w$ is continuous in $\bar{D}_{\tau}$ and if it satisfies $L w \leqq 0$ in $D_{\tau}, w \geqq 0$ on $B$ and $\partial w / \partial T>0$ on $S_{r}$, then $w \geqq 0$ in $\bar{D}_{\tau}$.

Theorem 1. Let L satisfy (A), (B) and let $g(x, t, u)$ satisfy $\left(\mathrm{G}_{2}\right)$. For every $\tau$ there exists at most one solution $u(x, t)$ in $D_{\tau}$ of the system (1.1), (1.2), (1.3) which is continuous in $\bar{D}_{\tau}$.

Proof. Suppose $u$ and $v$ are two solutions and write $w=u-v$. Then $L w=0$ and

$$
\frac{\partial w}{\partial T}=g(x, t, u)-g(x, t, v) .
$$

If $w \neq 0$ then $w$ must assume either positive or negative values in $D_{\tau}$. It will be enough to consider the case that $w$ assumes negative values. In that case $w$ assumes a negative minimum in $\bar{D}_{r}$, say at a point $P$. By Lemma $1 P$ belongs to $S_{r}$ and $\partial w / \partial T \leqq 0$ at $P$. On the other hand, $u<v$ at $P$ and using (1.5) and the property $\left(\mathrm{G}_{2}\right)$ of $g$ we conclude that $\partial w / \partial T>0$ at $P$, which is a contradiction.

Theorem 2. Let $L$ satisfy (A), (B) and let $g(x, t, u)$ satisfy $\left(\mathrm{G}_{2}\right),\left(\mathrm{G}_{3}\right)$. If $u$ is a solution in $D_{r}$ of the system (1.1), (1.2), (1.3) and if it is continuous in $\bar{D}_{\tau}$, then

$$
|u(x, t)| \leqq \max (1, \underset{x \in B}{\operatorname{lu} . b .}|\psi(x)|) ;
$$

more precisely, (a) for $t>0, u>\min (0$, g.l.b. $\psi(x))$; (b) if l.u.b. $\psi(x) \leqq 1$ then $u \leqq 1$; (c) if l.u.b. $\psi(x)>1$ then for $t>0, u<$ l.u.b. $\psi(x)$. The above statements remain true if we replace both of the inequality signs $>,<$ by $\geqq$,

Proof. If (a) is not true then $u$ must take in $D_{\tau}$ nonpositive values which are $\leqq$ g.l.b. $\psi(x)$. Thus $u$ must assume its nonpositive minimum in $D_{\tau}$ and by the strong maximum principle that minimum is assumed at a point $P$ on $S_{\tau}$. We clearly have $\partial u / \partial T \leqq 0$ at $P$. Hence, by (1.3),

$$
g(P, u(P))=\frac{\partial u}{\partial T} \leqq 0 .
$$


On the other hand, using $\left(\mathrm{G}_{2}\right),\left(\mathrm{G}_{3}\right)$ and the fact that $u(P) \leqq 0$ we get $g(P, u(P)) \geqq g(P, 0)>0$ which is a contradiction.

To prove (b) assume on the contrary that $u$ takes values $>1$. Then by the strong maximum principle $u$ assumes its maximum in $\bar{D}_{\tau}$ at a point $P$ on $S_{\boldsymbol{r}}$ and $u(Q)<u(P)$ for all $Q$ in $D_{r}$.

We then have $\partial u / \partial T \geqq 0$ at $P$. Hence, by (1.3),

$$
g(P, u(P))=\frac{\partial u}{\partial T} \geqq 0 .
$$

On the other hand, using $\left(\mathrm{G}_{2}\right),\left(\mathrm{G}_{3}\right)$ and the fact that $u(P)>1$ we get

$$
g(P, u(P))<g(P, 1) \leqq 0
$$

which is a contradiction.

The proof of (c) is similar to the proof of (b) and will be omitted. We also omit the proof of the last assertion of the theorem.

Remark 1. If $c(x, t) \equiv 0$ in (1.1) and if $g(x, t, u)>0$ for each $u<1$ then we can prove a statement more precise than (a), namely, (a') if g.l.b. $\psi(x)<1$ $(\geqq 1)$ then, for $t>0, u>$ g.l.b. $\psi(x)(\geqq 1)$.

Remark 2. If $\psi(x) \equiv 0$ then Theorem 2 says that $0<u(x, t) \leqq 1$ for $t>0$. If, however, $\tau=\infty$ in Theorem 2 then on using [3; Theorem 2] we can prove that $0<u(x, t)<1$ for $t>0$.

By the method used to prove Theorem 2 we shall now prove the following lemma to be used later.

Lemma 2. Let L satisfy (A), (B) and let $g$ and $g^{\prime}$ satisfy $\left(\mathrm{G}_{2}\right)$. Let $u$ be a solution in $D_{\tau}$ of the system (1.1), (1.2), (1.3), let $u^{\prime}$ be a solution in $D_{\tau}$ of the system (1.1), (1.2), (1.3) with $g$ and $\psi$ replaced by $g^{\prime}$ and $\psi^{\prime}$ and assume that

$$
g^{\prime}(x, t, v) \geqq g(x, t, v), \quad \psi^{\prime}(x) \geqq \psi(x) .
$$

If $u$ and $u^{\prime}$ are continuous in $\bar{D}_{\tau}$, then $u^{\prime} \geqq u$ in $D_{\tau}$.

Proof. We have to show that the function $w=u-u^{\prime}$ is nonpositive in $D_{\tau}$. In the contrary case it follows on using Lemma 1 that $w$ assumes a positive maximum in $\bar{D}_{\tau}$ at a point $P$ on $S_{\tau}$ and $\partial w / \partial T \geqq 0$ at $P$. Noting, however, that $u(P)>u\left(P^{\prime}\right)$ and using $(1.7)$ we get

$$
\frac{\partial w}{\partial T}=g(P, u(P))-g^{\prime}\left(P, u^{\prime}(P)\right)<g\left(P, u^{\prime}(P)\right)-g^{\prime}\left(P, u^{\prime}(P)\right) \leqq 0
$$

which is a contradiction.

2. Existence Theorem. 2.1. In this section we replace (B) by the stronger assumption:

(B') The coefficients $a_{i j}, b_{i}$ and $c$ of $L$ are Hölder continuous in compact subsets of $\bar{D}$ and $c(x, t) \leqq 0$. 
We shall need the following assumption on $\psi(x)$ :

$(\Psi) \psi(x)$ is defined and continuous on $\bar{B}$ and has a compact support in $B$, that is, it vanishes near the boundary $\dot{B}$.

Theorem 3. Let $L$ satisfy the assumptions (A), $\left(\mathrm{B}^{\prime}\right)$, let $g(x, t, u)$ satisfy the assumptions $\left(\mathrm{G}_{1}\right),\left(\mathrm{G}_{2}\right),\left(\mathrm{G}_{3}\right)$ and let $\psi(x)$ satisfy the assumption $(\Psi)$. Then there exists a solution in $D$ of the system (1.1), (1.2), (1.3) which is continuous in $\bar{D}$.

Remark. In [5] we gave a proof of this theorem in the special case of the heat equation. In that proof we made extensive use of generalized simple layers and our arguments can easily be generalized to equations (1.1) with sufficiently regular coefficients (so that the fundamental solutions constructed by DrEsSEL [1], [2] exist). Since then we found that fundamental solutions and simple layers have been thoroughly studied by Pogonzelski [11], [12] under the very weak assumptions (A), $\left(\mathrm{B}^{\prime}\right)$ on the coefficients of $L$. He has also proved existence of solutions for some nonlinear boundary value problems [10], [13], [14]. In particular, he solved in $D_{\tau}$ the system (1.1), (1.2), (1.3) with $\psi$ any continuous function (and with $\bar{B}$ replaced by $B$ in (1.2)) provided $g(x, t, u)(-\infty<u<\infty$, $0 \leqq t \leqq \tau, x \varepsilon \bar{B})$ is continuous and bounded. His solutions need not be continuous or even bounded near $\dot{B}$ and thus uniqueness is not guaranteed. Without uniqueness we cannot continue solutions defined in each $D_{\tau}$ into a solution in $D$. Below we give a simplification of his method using our results of $\$ 1$.

Proof. We first prove existence of a solution in each $D_{\sigma}$. Let $\Gamma(x, t ; \xi, \tau)$ be a fundamental solution in $D_{\sigma}$ of equation (1.1) as constructed in [11], and define

$$
\varphi(x, t)=\int_{B} \rho(\xi, 0) \Gamma(x, t ; \xi, 0) \psi(\xi) d \xi
$$

where

$$
\rho(\xi, \tau)=\left(\operatorname{det} a^{i j}(\xi, \tau)\right)^{\frac{1}{2}} / 2^{n} \pi^{\frac{1}{2} n}
$$

and $\left(a^{i j}\right)$ is the matrix inverse to $\left(a_{i j}\right)$. As was proved in [12], $\varphi(x, t)$ is a solution of $(1.1)$ in $D_{\sigma}$, continuous in $\bar{D}_{\sigma}$, and $\varphi(x, 0)=\psi(x)$. We also observe that

$$
\frac{\partial \varphi}{\partial T} \text { is continuous on } \overline{S_{\sigma}}
$$

(here we make use of the assumption $(\Psi)$ ).

Denote by $Z$ the Banach space of all functions $u(x, t)$ continuous in $\bar{D}_{\sigma}$ with the norm

$$
\|u\|=\underset{D_{\sigma}}{1 . u . b .}|u(x, t)|
$$

and denote by $Z_{R}$ the set $\{u ; u \varepsilon Z,\|u\|<R\}$. We define a transformation $v=T u$ for $u \varepsilon Z_{R}$ in the following manner: $v$ is a solution of (1.1) in $D_{\sigma}$, con- 
tinuous in $\bar{D}_{\sigma}$, and it satisfies $v=\psi$ on $\bar{B}$ and $\partial v / \partial T=g(x, t, u)$ on $S_{\sigma}$. By Theorem $1, v$ is uniquely determined. We try to find it in the form

$$
v(x, t)=\int_{0}^{t} \int_{\dot{B}} 2 \rho(\xi, \tau) \Gamma(x, t ; \xi, \tau) h(\xi, \tau) d W_{\xi} d \tau+\varphi(x, t)
$$

where $d W_{\xi}$ is the element of surface on $\dot{B}$ at the point $\xi, \rho(\xi, \tau)$ is defined by (2.2) and $h$ is still to be determined. The integral in (2.4) is a generalized simple layer with density $h$. Using [12] we conclude: $v$ is a solution of the equation (1.1) in $D_{\sigma}$, it is continuous in $\bar{D}_{\sigma}$, it satisfies $v(x, 0)=\psi(x)$ on $\bar{B}$ and

$$
\begin{aligned}
\left.\frac{\partial v}{\partial T}\right|_{(x, t)}=h(x, t)+\int_{0}^{t} \int_{\dot{B}} 2 \rho(\xi, \tau) \frac{\partial}{\partial T} \Gamma(x, t & ; \xi, \tau) h(\xi, \tau) d W_{\xi} d \tau \\
& +\left.\frac{\partial \varphi}{\partial T}\right|_{(x, t)} \quad\left((x, t) \varepsilon S_{\sigma}\right) .
\end{aligned}
$$

Putting $\partial v / \partial T=g(x, t, u)$ we obtain for $h$ the integral equation

$$
\begin{aligned}
h(x, t)+\int_{0}^{t} \int_{\dot{B}} 2 \rho(\xi, \tau) \frac{\partial}{\partial T} \Gamma(x, t ; \xi, \tau) h(\xi, \tau) d W_{\xi} d \tau & \\
& =g(x, t, u(x, t))-\left.\frac{\partial \varphi}{\partial T}\right|_{(x, t)} .
\end{aligned}
$$

Since the kernel is integrable [12], this singular Volterra type integral equation can be solved (and uniquely) by successive iterations (compare [11; pp. 41-44]). We then get that $h(x, t)$ is continuous on $\vec{S}_{\sigma}$ and

$$
\underset{S_{\sigma}}{\text { l.u.b. }}|h(x, t)| \leqq A+\underset{S_{\sigma}}{\text { l.u.b. }}|g(x, t, u(x, t))|
$$

where $A$ is a constant independent of $u$.

By Theorem 2 any possible solution is bounded by $\lambda \equiv \max (1$, l.u.b. $|\psi|)$. It follows that if we define

$$
G(x, t, u)=\left\{\begin{array}{lll}
g(x, t, u) & \text { if } & |u| \leqq \lambda \\
g(x, t, \pm \lambda) & \text { if } \quad \pm u>\lambda
\end{array}\right.
$$

and prove the existence of a solution $u$ of (1.1), (1.2), (1.3) with $g$ replaced by $G$ then $u$ will also be a solution of the original problem. Since $G$ is bounded for $(x, t) \varepsilon D_{\sigma}$ and $-\infty<u<\infty$, we conclude that without loss of generality we may assume from the beginning that

$$
|g(x, t, u)| \leqq A_{1} \quad((x, t) \varepsilon D,-\infty<u<\infty)
$$

where $A_{1}$ is a constant.

Substituting (2.8) into (2.7) we conclude that for every $u \varepsilon Z_{R}$

$$
\underset{s}{\text { l.u.b. }}|h(x, t)| \leqq A+A_{1} \text {. }
$$


Substituting (2.9) into (2.4) we get

$$
\underset{D \sigma}{\text { l.u.b. }}|v(x, t)| \leqq K
$$

where $K$ is a constant independent of $R$ and of the particular $u$ in $Z_{R}$. Taking $R \geqq K$ it follows that $v=T u$ maps $Z_{R}$ into itself.

To prove that $v=T u$ is a continuous transformation let $u_{m} \varepsilon Z_{R},\left\|u_{m}-u\right\| \rightarrow 0$ as $m \rightarrow \infty$ and denote by $h_{m}$ the corresponding solutions of (2.6). It then follows that

$$
\text { l.u.b. }\left|h_{m}-h\right| \leqq \text { const. l.u.b. }\left|g\left(x, t, u_{m}\right)-g(x, t, u)\right|
$$

and by $\left(\mathrm{G}_{1}\right)$ the right side tends to zero as $m \rightarrow \infty$. Denoting $v_{m}=T u_{m}$ and using (2.4) with $h, v$ replaced by $h_{m}, v_{m}$ we conclude that $\left\|v_{m}-v\right\| \rightarrow 0$ as $m \rightarrow \infty$ and the continuity of $T$ is thereby proved.

We shall now prove that $T$ maps $Z_{R}$ into a compact set in $Z$. The functions

$$
\int_{0}^{t} \int_{\dot{B}} 2 \rho(\xi, \tau) \Gamma(x, t ; \xi, \tau) h(\xi, \tau) d W_{\xi} d \tau, \quad u \varepsilon Z_{R}
$$

satisfy a uniform Hölder condition with any exponent $\vartheta<1$ in $x$ and $\frac{1}{2} \vartheta$ in $t$. This follows from [12] and (2.9). Hence the set of functions (2.10) is a compact set in $Z$. If we add to each function in (2.10) the function $\varphi(x, t)$ (which is continuous in $\bar{D}_{\sigma}$ ) then clearly the resultant set of functions is also a compact subset of $Z$. Thus the set $\left\{v ; v=T u, u \varepsilon Z_{R}\right\}$ is compact.

Having proved that $T$ is a continuous transformation which maps the closed bounded convex set $Z_{R}$ of the Banach space $Z$ into a compact subset of itself, we can apply the Schauder fixed point theorem [16] and thus conclude that there exists $u \varepsilon Z_{R}$ for which $u=T u$. This proves the existence of a solution in $D_{\sigma}$.

We now note that if $u_{\sigma}$ and $u_{r}$ are solutions of (1.1), (1.2), (1.3) in $D_{\sigma}$ and $D_{\tau}$ respectively and if $\sigma \geqq \tau$ then $u_{\sigma} \equiv u_{\tau}$ in $D_{\tau}$. Indeed, this follows from Theorem 1 . Using this remark we define a unique solution in $D$ in the following way: In each $D_{\sigma}, u=u_{\sigma}$ where $u_{\sigma}$ is the solution of (1.1), (1.2), (1.3) in $D_{\sigma}$.

2.2. The assumption $(\Psi)$ was needed in order to conclude (2.3). However, if the coefficients of $L$ satisfy additional regularity conditions then we can weaken the assumption $(\Psi)$. We shall make the following assumption:

$\left(\mathrm{B}^{\prime \prime}\right)$ The coefficients of $L$ are defined and Hölder continuous in compact subsets of the closure of a cylinder $D^{\prime}$ with base $B^{\prime}$ which contains $\bar{B}$, and they have first continuous $x$-derivatives in some $D^{\prime}$-neighborhood of $\dot{B}$. Furthermore, the functions $\partial a_{i j} / \partial x_{\lambda}$ are Hölder continuous (in $(x, t)$ ) in that $D^{\prime}$-neighborhood. Finally, $c(x, t) \leqq 0$.

By a $D^{\prime}$-neighborhood of $\dot{B}$ we mean a set of points $(x, t)$ which satisfy $(x, t) \varepsilon \overline{D^{\prime}}$, distance from $x$ to $\dot{B}$ is $\leqq \epsilon$ and $t \leqq \epsilon$ for some $\epsilon>0$.

We shall need the following assumption (weaker then $(\Psi)$ ):

$\left(\Psi^{\prime}\right) \psi(x)$ is continuous in $\overline{B^{\prime}}$ and it is continuously differentiable in some neighborhood of $\dot{B}$. 
We can now prove

Theorem 3*. Theorem 3 remains true if we replace $\left(\mathrm{B}^{\prime}\right)$ and $(\Psi)$ by $\left(\mathrm{B}^{\prime \prime}\right)$ and $\left(\Psi^{\prime}\right)$.

Proof. We denote by $\Gamma(x, t ; \xi, \tau)$ the fundamental solution of Pogonzelski constructed in $D_{\sigma}^{\prime}$ instead of $D_{\sigma}$. Then the proof of Theorem $3^{*}$ proceeds as that of Theorem 3. If we prove (2.3) for the function

$$
\varphi(x, t)=\int_{B^{\prime}} \rho(\xi, 0) \Gamma(x, t ; \xi, 0) \psi(\xi) d \xi
$$

then the proof of Theorem $3^{*}$ is completed. For simplicity we shall first give the proof only for the case of the heat equation; the proof of the general case follows along the same lines if we make use of $[11 ; \mathrm{pp} .41-44]$. Since $\Gamma(x, t ; \xi, r)$ is differentiable in $x$ whenever $x \neq \xi$, it is enough to prove that the function

$$
\vartheta(x, t)=\int_{B^{\prime \prime}} \frac{\psi(\xi)}{t^{\frac{1}{2} n}} \exp \left\{-\frac{|x-\xi|^{2}}{4 t}\right\} d \xi
$$

is continuously $x$-differentiable in a small $D^{\prime}$-neighborhood of $\dot{B}$. Here $B^{\prime \prime}$ is any neighborhood of $\dot{B}$ where $\psi(x)$ is differentiable. We now differentiate $\vartheta(x, t)$ with respect to $x$ and integrate the resultant integral by parts. We obtain

$$
\frac{\partial \vartheta}{\partial x}=\int_{B^{\prime \prime}} \frac{\partial \psi(\xi)}{\partial \xi} \frac{1}{t^{\frac{1}{2} n}} \exp \left\{-\frac{|x-\xi|^{2}}{4 t}\right\} d \xi+\text { boundary integrals, }
$$

from which our assertion readily follows.

In the general case the function $\vartheta(x, t)$ in $(2.11)$ has to be replaced by (see [11])

$$
\begin{aligned}
& \int_{B^{\prime \prime}} \psi(\xi) w^{\xi, 0}(x, t ; \xi, 0) d \xi \\
& \quad+\int_{B^{\prime \prime}} \psi(\xi)\left[\int_{0}^{t} \int_{B^{\prime}} w^{y, \vartheta}(x, t ; y, \vartheta) \Phi(y, \vartheta ; \xi, 0) d y d \vartheta\right] d \xi .
\end{aligned}
$$

The first integral can be treated as in the previous case of the heat equation. In the second integral we may without loss of generality replace $B^{\prime}$ by $B^{\prime \prime}$. However, one cannot apply directly the previous method to the second integral with $B^{\prime}$ replaced by $B^{\prime \prime}$ since $\partial \Phi / \partial y$ and $\partial \Phi / \partial \xi$ have nonintegrable singularities at $\vartheta=0$. We therefore consider first the integral.

$$
I(\epsilon)=\int_{B^{\prime \prime}} \psi(\xi)\left[\int_{\epsilon}^{t} \int_{B^{\prime \prime}} w^{y, \vartheta}(x, t ; y, \vartheta) \Phi(y, \vartheta ; \xi, 0) d y d \vartheta\right] d \xi,
$$

where $\epsilon>0$. We have

$$
\frac{\partial I(\epsilon)}{\partial x}=\int_{B^{\prime \prime}} \psi(\xi)\left[\int_{\epsilon}^{t} \int_{B^{\prime}} \frac{\partial}{\partial x} w^{\nu, \vartheta}(x, t ; y, \vartheta) \Phi(y, \vartheta ; \xi, 0) d y d \vartheta\right] d \xi
$$


We now integrate by parts noting that

$$
\left(\frac{\partial}{\partial x}+\frac{\partial}{\partial y}\right) w^{y, \vartheta}(x, t ; y, \vartheta), \quad\left(\frac{\partial}{\partial y}+\frac{\partial}{\partial \xi}\right) \Phi(y, \vartheta ; \xi, 0)
$$

contribute terms of smaller order of magnitude. Here we make use of the assumption $\left(\mathrm{B}^{\prime \prime}\right)$ and of some formulas of $[11 ; \mathrm{pp} .41-44]$. We obtain

$$
\begin{gathered}
\frac{\partial I(\epsilon)}{\partial x}=\int_{B^{\prime \prime}} \frac{\partial \psi(\xi)}{\partial \xi}\left[\int_{\epsilon}^{t} \int_{B^{\prime \prime}} w^{y, \vartheta}(x, t ; y, \vartheta) \Phi(y, \vartheta ; \xi, 0) d y d \vartheta\right] d \xi \\
+\int_{B^{\prime \prime}} \psi(\xi)\left[\int_{\epsilon}^{t} \int_{B^{\prime \prime}} W d y d \vartheta\right] d \xi+\int_{B^{\prime \prime}} \psi(\xi)\left[\int_{\epsilon}^{t} \int_{\dot{B}^{\prime \prime}} R d S_{y} d \vartheta\right] d \xi
\end{gathered}
$$

where $\dot{B}^{\prime \prime}$ is the boundary of $B^{\prime \prime}, d S_{y}$ is the surface element of $B^{\prime \prime}$ at $y$ and $R$, $W$ are appropriate expressions (when $\epsilon=0$ the singularity of $W$ is absolutely integrable).

Comparing (2.13) with (2.14) and taking $\epsilon \rightarrow 0$, and then noting that the two last integrals on the right side of (2.14) are continuous functions in a $D^{\prime}$-neighborhood of $\dot{B}$ when $\epsilon=0$, the proof is completed.

2.3. The assumptions on $\psi$ can also be relaxed if we make more assumptions on $g$. We shall need the following assumption on $g$ :

$\left(\mathrm{G}_{4}\right)$ There exists a positive constant $A$ such that $g(x, t, u)$ satisfies the Lipschitz condition (in $u$ )

$$
\left|g\left(x, t, u^{\prime}\right)-g\left(x, t, u^{\prime \prime}\right)\right| \leqq A\left|u^{\prime}-u^{\prime \prime}\right|
$$

for all $(x, t) \varepsilon \bar{D}$ and $\left|u^{\prime}\right|,\left|u^{\prime \prime}\right| \leqq \max (1$, l.u.b. $|\psi|)$.

We can now prove the following theorem:

Theorem $\overline{3}$. Let $L$ satisfy the assumptions $(\mathrm{A}),\left(\mathrm{B}^{\prime}\right)$, let $g(x, t, u)$ satisfy the assumptions $\left(\mathrm{G}_{1}\right),\left(\mathrm{G}_{2}\right),\left(\mathrm{G}_{3}\right),\left(\mathrm{G}_{4}\right)$ and let $\psi(x)$ be a continuous function on $\bar{B}$. Then there exists a solution in D of the system (1.1), (1.2), (1.3) which is continuous in $\bar{D}$.

Proof. We first construct a solution in $D_{\sigma}$, for $\sigma$ sufficiently small. The method we use is that of successive approximation. We define $v_{1}(x, t)=\varphi(x)$ and then define $v_{m+1}(x, t)$ in terms of $v_{m}(x, t)$ as follows: Let $h=h_{m+1}$ be a solution of (2.6) with $u(x, t)$ replaced by $v_{m}(x, t)$. Then substitute $h=h_{m+1}$ in (2.4) and let $v=v_{m+1}$.

If we use the inequalities

$$
\begin{gathered}
\left|\frac{\partial \varphi(x, t)}{\partial T}\right| \leqq \frac{\text { const. }}{t^{\gamma}} \\
|\Gamma(x, t ; \xi, \tau)| \leqq \frac{\text { const. }}{(t-\tau)^{\mu}} \frac{1}{|x-\xi|^{n-2 \mu}}
\end{gathered}
$$


for $\frac{1}{2}<\gamma<\mu<1$, then we can show by induction that

$$
\text { l.u.b. }\left|v_{m+1}(x, t)-v_{m}(x, t)\right| \leqq K^{m} t^{(1-\mu) m} \quad(m=1,2, \cdots)
$$

for all $0<t \leqq \sigma$, where the constant $K$ is an increasing function of $\sigma$. Here we have made use of $\left(\mathrm{G}_{4}\right) \cdot h_{m+2}-h_{m+1}$ is also bounded by const. $t^{(1-\mu) m}$. (Actually we first modify the definition of $g$ for $|u|>\max (1$, l.u.b. $|\psi|)$ so as to get a new function which satisfies the Lipschitz condition for all u.) From (2.15) it follows that for $\sigma$ sufficiently small the sequence $\left\{v_{m}\right\}$ converges to a solution $u(x, t)$.

We can now repeat the above argument and construct step by step a solution $u$ in the whole cylinder $D$. Here we make use of Theorem 1 .

3. Further Properties of Solutions. In this section we shall prove some properties of the solution of the system (1.1), (1.2), (1.3). The first theorem is concerned with the existence of $\lim u(x, t)$ as $t \rightarrow \infty$. We shall need the following assumptions:

$\left(\mathrm{G}_{3}^{\prime}\right) g(x, t, 1) \equiv 0$.

$\left(\mathrm{G}_{4}^{\prime}\right)$ There exist positive constants $\eta, A$ such that for all $(x, t) \varepsilon \bar{D}$ and for all $u^{\prime}>u^{\prime \prime}$ bounded by $\max (1$, l.u.b. $|\psi|)$

$$
-A\left(u^{\prime}-u^{\prime \prime}\right) \leqq g\left(x, t, u^{\prime}\right)-g\left(x, t, u^{\prime \prime}\right) \leqq-\eta\left(u^{\prime}-u^{\prime \prime}\right) .
$$

(C) There exist positive constants $K_{1}, K_{2}$ such that

$$
a_{11}(x, t) \geqq K_{1}>0, \quad-b_{1}(x, t) \leqq K_{2} \quad \text { for all }(x, t) \varepsilon D .
$$

We shall prove the following theorem:

Theorem 4. Let L satisfy (A), ( $\left.\mathrm{B}^{\prime}\right),(\mathrm{C})$, let $c(x, t) \equiv 0$ and let $g$ satisfy $\left(\mathrm{G}_{1}\right)$, $\left(\mathrm{G}_{2}\right),\left(\mathrm{G}_{3}^{\prime}\right),\left(\mathrm{G}_{4}^{\prime}\right)$. If $u(x, t)$ is a solution of (1.1), (1.2), (1.3) in D and is continuous in $\bar{D}$, then $\lim _{t \rightarrow \infty} u(x, t)=1$ uniformly in $x \in \bar{B}$. More precisely, there exists a positive constant $\mu$ such that

$$
|1-u(x, t)|=O(\exp \{-\mu t\})
$$

uniformly in $x \in \bar{B}$, as $t \rightarrow \infty$.

Proof. We first consider the special case that l.u.b. $\psi \leqq 1$. In that case we know, by Theorem 2 , that $u(x, t) \leqq 1$. The function $v=1-u$ is nonnegative in $D$ and satisfies $L v=0$ in $D$ and

$$
\begin{aligned}
\frac{\partial v}{\partial T}+\eta v=- & g(x, t, u)+\eta v \\
& =g(x, t, 1)-g(x, t, u)+\eta v \leqq-\eta(1-u)+\eta v=0 .
\end{aligned}
$$

Here we made use of $\left(G_{4}^{\prime}\right)$.

Consider now the function

$$
w=e^{\alpha R}-e^{\alpha x_{1}} .
$$


If $R$ is sufficiently large, then $w>0$ in $\bar{B}$. Using (C) it follows that we can fix $\alpha$ (independently of $R$ ) such that

$$
L w=\left(-\alpha^{2} a_{11}-\alpha b_{1}\right) e^{\alpha x_{1}}+c w \leqq-e^{\alpha x_{1}} .
$$

We now choose $R$ sufficiently large such that also

$$
\frac{\partial w}{\partial T}+\eta w=-\alpha e^{\alpha x_{1}} \frac{\partial x_{1}}{\partial T}+\eta\left(e^{\alpha R}-e^{\alpha x_{1}}\right)>0 \text { on } \dot{B}
$$

and put

$$
\lambda=\min _{\bar{B}} \frac{e^{\alpha x_{1}}}{e^{\alpha R}-e^{\alpha x_{1}}}>0 .
$$

From (3.4), the definition (3.3) and (3.6) it follows that the function

$$
z(x, t)=e^{-\lambda t} w(x)
$$

satisfies $L z \leqq 0$. From (3.5) it follows that

$$
\frac{\partial z}{\partial T}+\eta z>0 \text { on } S .
$$

Taking $K$ to be such that $K z(x, 0)>u(x, 0)$, we shall prove that

$$
v(x, t) \leqq K z(x, t)=K e^{-\lambda t}\left(e^{\alpha R}-e^{\alpha x_{1}}\right)
$$

from which (3.1) would follow.

To prove (3.9) define $y=v-K z$. Using (3.2), (3.8) it follows that

$$
\frac{\partial y}{\partial T}+\eta y<0 \text { on } S \text {. }
$$

Furthermore, $L y \geqq 0$ in $D$ and $y \leqq 0$ on $\bar{B}$. We have to prove that $y \leqq 0$ in $D$. In the contrary case, $y$ assumes positive values in $D$ and therefore, for some $\sigma>0, y$ has a positive maximum in $\bar{D}$ which, by the maximum principle, is assumed at a point $P$ on $S_{\sigma}$. At that point $\partial y / \partial T \geqq 0$ and $\eta y>0$. Thus $\partial y / \partial T+$ $\eta y>0$ at $P$ which contradicts (3.10).

Having proved Theorem 4 in case l.u.b. $\psi \leqq 1$, we remark that a similar proof can be constructed in the case where g.l.b. $\psi>1$. We have only to define $v=u-1$ and then proceed as before.

To prove Theorem 4 for general functions $\psi(x)$ we introduce two constant functions

Then

$$
\psi_{1}(x) \equiv \min (0, \text { g.l.b. } \psi), \quad \psi_{2}(x) \equiv \max (1, \text { l.u.b. } \psi) .
$$

and

$$
\psi_{1}(x) \leqq \psi(x) \leqq \psi_{2}(x) \text { for } \quad x \varepsilon \bar{B}
$$

$$
\psi_{1}(x) \leqq 1 \leqq \psi_{2}(x) \text { for } x \varepsilon \bar{B}
$$


Since $\psi_{1}$ and $\psi_{2}$ are continuous functions, there exist by Theorem $\overline{3}$ solutions $u_{1}$ and $u_{2}$ of the system (1.1), (1.2), (1.3) with $\psi$ replaced by $\psi_{1}$ and $\psi_{2}$ respectively. From Lemma 2 it follows that

$$
u_{1}(x, t) \leqq u(x, t) \leqq u_{2}(x, t) .
$$

By what we have already proved

$$
1-u_{i}(x, t)=O\left(\exp \left\{-\lambda_{i} t\right\}\right) \text { as } t \rightarrow \infty \quad\left(\lambda_{i}>0\right) .
$$

Using (3.14) in (3.13), the proof of (3.1) is completed.

Remark. In $\$ 5$ we shall see that Theorem 4 remains true if the assumption $c(x, t) \equiv 0$ is replaced by the weaker assumption $\lim c(x, t)=0(t \rightarrow \infty)$.

The next theorem considers the question of monotonicity of $u$ in $t$. We shall need the following assumption on $g$ :

$\left(\mathrm{G}_{5}\right) g(x, t, u)$ is a non-decreasing function of $t$.

Theorem 5. Let the coefficients of $L$ satisfy (A), (B) and be independent of $t$. Let $g$ satisfy $\left(\mathrm{G}_{2}\right),\left(\mathrm{G}_{3}\right),\left(\mathrm{G}_{5}\right)$. If $u(x, t)$ is continuous in $\bar{D}$ and satisfies the system (1.1), (1.2), (1.3) and if $\psi(x) \equiv$ const. $<1$ and $\geqq 0$ then $u(x, t)$ is nondecreasing in $t$.

Proof. Let $v(x, t)=u(x, t+h)-u(x)$ where $h$ is an arbitrary positive constant. Then $L v=0$ in $D$ and, by Theorem $2, v$ is positive on $t=0$. If $v$ is not zero or positive at each point of $D$, then, for some $\sigma>0, v$ assumes a negative minimum in $\bar{D}_{\sigma}$. From Lemma 1 we conclude that the minimum is assumed at a point $P$ on $S_{\sigma}$ and $\partial v / \partial T \leqq 0$ at $P$. On the other hand, using $\left(\mathrm{G}_{2}\right),\left(\mathrm{G}_{5}\right)$ we have

$$
\frac{\partial v}{\partial T}=g(x, t+h, u(x, t+h))-g(x, t, u(x, t))>0
$$

at $P$, since $u(x, t+h)<u(x, t)$ at $P$. We have thus arrived at a contradiction which proves the theorem.

Remark 1. Theorem 5 remains true for solutions $u(x, t)$ with $\psi(x) \equiv$ const. $<0$ provided $c(x, t) \equiv 0$.

Remark 2. A similar theorem can be proved if $\psi(x) \equiv$ const. $>1$. If $\psi(x) \equiv$ const. Theorem 5 need not be true. Take, for instance, the heat equation and assume that $\psi\left(x^{0}\right)<1\left(x^{0} \varepsilon B\right)$ and that $\psi(x)$ decreases very fast as $\left|x-x^{0}\right|$ increases. Since $u$ has the physical meaning of the heat distribution (see Introduction) it is clear (from physical experience-and it can also be checked mathematically) that $u\left(x^{0}, t\right)$ does not increase monotonically in $t$.

Remark 3. Using [3; Theorem 2] we can prove, under the assumptions of Theorem 5 , that $u(x, t)$ is strictly increasing in $t$.

4. Varing Temperature of the Gas. In the previous sections we considered generalized heat transfer problems under the condition that the temperature of 
the gas is 1 , that is, $g(x, t, 1) \equiv 0$ (sometimes the weaker condition $g(x, t, 1) \leqq 0$ ). In this chapter we shall mainly be concerned with generalized heat transfer problems in the case that the gas temperature varies with time. We shall consider two different situations:

(a) The temperature of the gas is a function $\varphi(t)$ of the time $t$;

(b) The temperature of the gas depends on $t$ and on the temperature $u$ of the body.

Although the situation (a) might very well occur in nature, the situation (b) is much more common. The precise a priori dependence of the gas temperature (near the body) on $u$ should be very complicated and we shall consider here an extremely simple model which perhaps represents a typical situation.

4.1. Problem (a). To discuss the problem (a), we assume that a function $g(x, t, u, v)((x, t) \varepsilon \bar{S},-\infty<u, v<\infty)$ is given, and we shall make use of the following assumptions:

$\left(\overline{\mathrm{G}}_{1}\right) g(x, t, u, v)$ is continuous for $(x, t) \varepsilon \bar{S},-\infty<u, v<\infty$.

$\left(\overline{\mathrm{G}}_{2}\right) g(x, t, u, v)$ is (strictly) decreasing in $u$ and (strictly) increasing in $v$.

$\left(\overline{\mathrm{G}}_{3}\right) g(x, t, u, u) \leqq 0$ and $g(x, t, u-\alpha, u)>0$ for some $\alpha>0$ and for all $(x, t) \varepsilon \bar{S},-\infty<u<\infty$.

$\left(\overline{\mathrm{G}}_{4}\right)$ There exists a positive constant $A$ such that

$$
\left|g\left(x, t, u^{\prime}, v\right)-g\left(x, t, u^{\prime \prime}, v\right)\right| \leqq A\left|u^{\prime}-u^{\prime \prime}\right|
$$

for all $(x, t) \varepsilon \bar{D},|v| \leqq$ l.u.b. $|\varphi(t)|$ and $\left|u^{\prime}\right|,\left|u^{\prime \prime}\right| \leqq \max (1$, l.u.b. $|\psi|)$.

In what follows we shall assume that $\varphi(t)$, the gas temperature, is a continuous function of $t$. We now replace (1.3) by

$$
\frac{\partial u}{\partial T}=g(x, t, u, \varphi(t))
$$

and proceed to discuss the extensions of Theorems 1-5.

Theorem 1'. Theorem 1 remains true for solutions of the system (1.1), (1.2), (4.1) ( $g$ is assumed only to satisfy $\left(\overline{\mathrm{G}}_{2}\right)$ ).

The proof follows word by word the proof of Theorem 1 .

Theorem $2^{\prime}$. Assume that $g$ satisfies $\left(\overline{\mathrm{G}}_{2}\right),\left(\overline{\mathrm{G}}_{3}\right)$ and that $L$ satisfies (A), (B). If $u$ is a solution of (1.1), (1.2), (4.1) in $D_{\tau}$ and is continuous in $\bar{D}_{\tau}$, then

$$
|u(x, t)| \leqq \max (\underset{0<t<\tau}{\text { l.u.b. }} \varphi(t), \underset{0<t<\tau}{\text { g.l.b. }}(\varphi(t)-\alpha), \underset{x \in B}{\text { l.u.b. }}|\psi(x)|) .
$$

One can easily formulate statements analogous to (a), (b), (c). We mention the following example: If $c(x, t) \equiv 0$ and $\psi(x) \leqq \varphi(t)$ for all $x \varepsilon \bar{B}$ and $t \geqq 0$ then

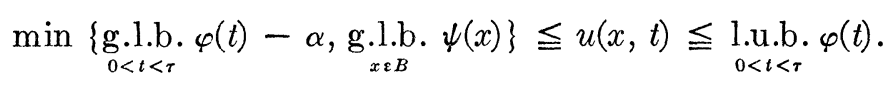


In particular, if $\psi(x) \equiv A \leqq \varphi(0)$ and if $\varphi(t)$ increases monotonically with $t$, then $\min (A, \varphi(0)-\alpha) \leqq u(x, t) \leqq \varphi(t)$.

The proof of Theorem $2^{\prime}$ can be carried out along the lines of proof of Theorem 2 and will be omitted.

Lemma $2^{\prime}$. Let $L$ satisfy (A), (B) and let $g$ satisfy $\left(\overline{\mathrm{G}}_{2}\right)$. Let $u$ be a function in $D_{r}$ satisfying (1.2), (4.1) and let $u^{\prime}$ be a function in $D_{r}$ satisfying the same system but with $\varphi^{\prime}$ and $\psi^{\prime}$ replacing $\varphi$ and $\psi$. If $\varphi^{\prime} \geqq \varphi, \psi^{\prime} \geqq \psi$ and if $u$ and $u^{\prime}$ are continuous in $\bar{D}_{\tau}$ and $L u^{\prime} \leqq L u$ in $D_{r}$, then $u^{\prime} \geqq u$ in $D_{\tau}$.

The proof of the lemma will be omitted.

With the aid of Theorems $1^{\prime}, 2^{\prime}$ we can now proceed to prove the analogues of Theorems $3,3^{*}$ and $\overline{3}$ for the system (1.1), (1.2), (4.1), noting (for instance, in proving the analogue of Theorem 3) that as in the proof of Theorem 3 (see (2.8)) we may assume without loss of generality that in each $D_{a}$

$$
\mid g\left(x, t, u, \varphi(t) \mid \leqq A_{1}\right.
$$

where $A_{1}$ is independent of $R$ and of the specific $u$ in $Z_{R}$. We thus conclude

Theorem $3^{\prime}$. Theorems $3,3^{*}$ (and $\overline{3}$ ) remain true for this system (1.1), (1.2), (4.1) with $g(x, t, u, v)$ satisfying $\left(\overline{\mathrm{G}}_{1}\right),\left(\overline{\mathrm{G}}_{2}\right),\left(\overline{\mathrm{G}}_{3}\right)$ (and $\left(\overline{\mathrm{G}}_{4}\right)$ ) and $\varphi(t)$ continuous.

To generalize Theorem 4 we shall assume that

$$
\varphi(\infty)=\lim _{t \rightarrow \infty} \varphi(t) \quad \text { exists. }
$$

We shall also need the following assumptions:

$\left(\overline{\mathrm{G}}_{3}^{\prime}\right) g(x, t, u, u)=0$ for all $u$ and all $(x, t) \varepsilon \bar{D}$.

$\left(\overline{\mathrm{G}}_{4}^{\prime}\right)$ There exist positive constants $\eta, A$ such that for all $(x, t) \varepsilon \bar{D}$, for all $u^{\prime}>u^{\prime \prime}$ bounded by $\max (1$, l.u.b. $|\psi(x)|)$ and for all $v$ bounded by l.u.b. $|\varphi(t)|$,

$$
-A\left(u^{\prime}-u^{\prime \prime}\right) \leqq g\left(x, t, u^{\prime}, v\right)-g\left(x, t, u^{\prime \prime}, v\right) \leqq-\eta\left(u^{\prime}-u^{\prime \prime}\right) .
$$

Theorem $4^{\prime}$. Let L satisfy $(\mathrm{A}),\left(\mathrm{B}^{\prime}\right),(\mathrm{C})$ and let $g$ satisfy $\left(\overline{\mathrm{G}}_{1}\right),\left(\overline{\mathrm{G}}_{2}\right),\left(\overline{\mathrm{G}}_{3}^{\prime}\right)$, $\left(\overline{\mathrm{G}}_{4}^{\prime}\right)$. If $\varphi(t)$ satisfies (4.4) and if $u(x, t)$ is a solution of (1.1), (1.2), (4.1) in $D$ which is continuous in $\bar{D}$, then

$$
\lim _{t \rightarrow \infty} u(x, t)=\varphi(\infty)
$$

uniformly with respect to $x$ in $\bar{B}$.

Proof. Let $\epsilon$ be an arbitrary positive number. We have to prove that

$$
|u(x, t)-\varphi(\infty)|<\epsilon \text { if } t \geqq t_{1}, x \varepsilon \bar{B} ;
$$

this will complete the proof of the theorem. Because of (4.4) we can find $\sigma$ such that

$$
\varphi(\infty)-\epsilon_{-} \leqq \varphi(t) \leqq \varphi(\infty)+\epsilon_{+} \quad \text { if } \quad t \geqq \sigma
$$

where $\epsilon_{+}=\min (\epsilon$, l.u.b. $\varphi(t)-\varphi(\infty)), \epsilon_{-}=\min (\epsilon, \varphi(\infty)-$ g.l.b. $\varphi(t))$. 
Denote by $u_{+}$the solution of the system

$$
\begin{aligned}
& L u_{+}=0 \text { in } D-D_{\sigma}, \\
& u_{+}(x, \sigma)=\underset{\xi \varepsilon B}{\operatorname{lup.b}} u(\xi, \sigma) \text { for } x \varepsilon \bar{B} \text {, } \\
& \frac{\partial u_{+}}{\partial T}=g\left(x, t, u_{+}, \varphi(\infty)+\epsilon_{+}\right) \text {on } S-S_{\sigma} .
\end{aligned}
$$

The existence of $u_{+}$follows from Theorem $\overline{3}$.

Using Lemma 2 ' we get

$$
u(x, t) \leqq u_{+}(x, t) \text { in } D-D_{\sigma} .
$$

We can apply Theorem 4 (with 1 replaced by $\varphi(\infty)+\epsilon_{+}$) to the function $u_{+}$ and thus get

$$
u_{+}(x, t)-\varphi(\infty)-\epsilon_{+}=O\left(\exp \left\{-\lambda_{1} t\right\}\right) \quad\left(\lambda_{1}>0\right) .
$$

Combining (4.7) with (4.8) we get

$$
u(x, t)<\varphi(\infty)+\epsilon_{+}+O\left(\exp \left\{-\lambda_{1} t\right\}\right) .
$$

In a similar manner we can prove that

$$
u(x, t)>\varphi(\infty)-\epsilon_{-}+O\left(\exp \left\{-\lambda_{2} t\right\}\right) \quad\left(\lambda_{2}>0\right) .
$$

Combining (4.9) with (4.10) and taking $t_{1}$ to be sufficiently large, the proof of (4.5) is completed.

Remark. The above proof can easily be modified to show that if $A_{1} \leqq$ $\varphi(t)<A_{2}$, then for any $\epsilon>0$ there exists $t_{1}$ such that $A_{1}-\epsilon \leqq u(x, t) \leqq A_{2}+\epsilon$ for $x \varepsilon \bar{B}, t>t_{1}$.

In extending Theorem 5 we shall clearly assume that $\varphi(t)$ is monotone in $t$, otherwise (as physical experience shows -it can also be checked mathematically) $u(x, t)$ need not be monotone in $t$. We shall need the following assumption:

$\left(\overline{\mathrm{G}}_{5}\right) g(x, t, u, v)$ is non-decreasing in $t$.

Theorem $5^{\prime}$. Let the coefficients of $L$ be independent of $t$ and satisfy (A), (B). Let $g$ satisfy $\left(\overline{\mathrm{G}}_{1}\right),\left(\overline{\mathrm{G}}_{2}\right),\left(\overline{\mathrm{G}}_{3}^{\prime}\right),\left(\overline{\mathrm{G}}_{5}\right)$. Finally, let $\varphi(t)$ be monotone increasing in $t$ and let $\psi(x)=$ const. $\leqq \varphi(0)$. If $u(x, t)$ is a solution of (1.1), (1.2), (4.1) and is continuous in $\bar{D}$, then it is non-decreasing in $t$.

Remark. Theorem $5^{\prime}$ remains true if $\left(\overline{\mathrm{G}}_{3}^{\prime}\right)$ is replaced by the weaker assumption $\left(\overline{\mathrm{G}}_{3}\right)$, provided $\alpha \leqq \varphi(0)-\psi(\psi$ being a constant function).

The proof of Theorem $5^{\prime}$ is similar to the proof of Theorem 5 via Theorem $2^{\prime}$ and will be omitted.

Remark. Theorems $1^{\prime}-5^{\prime}$ can easily be extended to the case that $\varphi=\varphi(x, t)$ is a continuous function in $(x, t)$ on $\bar{S}$. 
4.2. Problem (b). We shall consider the case where $\psi(x) \equiv 0$ and the following simple model of (b):

( $\Phi)$ The temperature of the gas at each point $x$ at the surface $\dot{B}$ of the body and at each time $t$ is given by

$$
\varphi=\varphi(t, u)=1-\rho(t) u
$$

where $u$ is the temperature of the body at $(x, t)$ and $\rho(t)$ is a continuous function of $t$ which satisfies:

$$
\rho(0)=0, \quad \rho(t) \geqq 0 \quad \text { for all } \quad t \geqq 0, \quad \lim _{t \rightarrow \infty} \rho(t)=0 .
$$

The assumption $(\Phi)$ corresponds to the case that the original temperature of the gas (before the body was inserted) was 1 . The cooling of the gas by the body at each point $(x, t)$ is $-\rho(t) u(x, t)$. When $t=0$ there is not yet any cooling $(\rho(0)=0)$ while as $t \rightarrow \infty$ we would expect the temperature $u$ to tend to that of the gas if the volume of the gas is large, so the cooling effect will reduce to zero $(\lim \rho(t)=0$ as $t \rightarrow \infty)$.

We shall consider the following system:

$$
\begin{gathered}
L u=0 \text { in } D, \\
u(x, 0)=0 \text { on } \bar{B}, \\
\frac{\partial u}{\partial T}=g(x, t, u, 1-\rho(t) u)
\end{gathered}
$$

where $g(x, t, u, v)$ is the function which appears in (4.1).

Using the fact that $\rho(t) \geqq 0$ we can easily extend Theorem $1^{\prime}$ to the present case.

To extend Theorem $2^{\prime}$ we first note that $u(x, t)>0$ if $t>0$. Indeed, in the contrary case we have a point $P$ on $S_{\tau}$ where $u$ takes its nonpositive minimum and where $\partial u / \partial T \leqq 0$. On the other hand, using (4.13), the properties $\left(\overline{\mathrm{G}}_{2}\right)$, $\left(\overline{\mathrm{G}}_{3}\right)$ with $\alpha=1$ and (4.12) we get

$$
\frac{\partial u}{\partial T}=g(x, t, u, 1-\rho(t) u)>0
$$

and we arrive at a contradiction.

In a similar way we can prove that $u(x, t) \leqq 1$ if $t>0$. Thus if $L$ satisfies (A), (B) and $g$ satisfies $\left(\overline{\mathrm{G}}_{2}\right),\left(\overline{\mathrm{G}}_{3}\right)$ with $\alpha=1$ and if $(\Phi)$ is satisfied, then any solution $u(x, t)$ of (4.13) in $D_{\tau}$ which is continuous in $\bar{D}_{\tau}$ must satisfy

$$
0<u(x, t) \leqq 1 \text { for } 0<t \leqq \tau, \quad x \varepsilon \bar{B} .
$$

If $\tau=\infty$ then we have the stronger inequality $u(x, t)<1$ in $\bar{D}$.

The proof of the last statement follows on using [3; Theorem 2].

Using the extensions of Theorems $1^{\prime}, 2^{\prime}$ which we have just proved we can 
proceed to extend Theorem $3^{\prime}$ to the system (4.13). The proof follows almost word by word the proof of Theorem $3^{\prime}$.

Theorem $4^{\prime}$ can also be very easily extended to the system (4.13). Indeed, since $0<u<1$,

$$
\varphi=\varphi(x, t)=1-\rho(t) u(x, t) \rightarrow 0 \text { as } t \rightarrow \infty
$$

uniformly in $x \in B$ and hence the proof of Theorem $4^{\prime}$ can be used to conclude that for every $\epsilon>0$

$$
0<1-u(x, t) \leqq \epsilon+O(\exp \{-\lambda t\}) \quad \text { if } \quad t \geqq t_{1}, \quad x \varepsilon \bar{B}
$$

where $t_{1}$ depends on $\epsilon$ and $\lambda>0$ is independent of $\epsilon$.

Theorem $5^{\prime}$ can also be extended to the present situation provided we assume that

$$
\text { if } u<w \text { then } g(x, t, u, 1-\rho(t) u)>g(x, t, w, 1-\rho(t) w)
$$

and that $g(x, t, u, v)$ is non-decreasing in $t$.

4.3. Partial Transfer of Heat. We shall briefly mention the case where heat transfer takes place only on a part $\dot{B}_{1}$ of $\dot{B}$ while the temperature on the complementary part $\dot{B}_{2}$ is given a priori; denote it by $k(x, t)$. We shall also make the assumption that $\dot{B}_{2}$ is a portion of an hyperplane, that $k$ vanishes near the boundary of $\dot{B}_{2}$, and that the coefficients of $L$ are defined and sufficiently smooth in a cylinder with the base $E$ to be defined below.

Theorems 1, 2 can easily be extended. To extend Theorem 3 we denote by $E$ an $n$-dimensional rectangle which contains $B$ and one of whose faces contains $\dot{B}_{2}$. Denote by $G(x, t ; \xi, \tau)$ the Green's function (constructed in [4]) corresponding to $L$ in the cylinder with base $E$. We then replace the right side of (2.4) in the proof of Theorem 3 by

$$
\begin{aligned}
\int_{0}^{t} \int_{\dot{B}_{1}} G(x, t ; \xi, \tau) h(\xi, \tau) & d W_{\xi} d \tau \\
& +\int_{0}^{t} \int_{\dot{B}_{2}} \frac{\partial}{\partial T} G(x, t ; \xi, \tau) k(\xi, \tau) d W_{\xi} d \tau+\varphi(x, t)
\end{aligned}
$$

where $\varphi$ is defined by (2.1) with $\Gamma$ replaced by $G$ and $\partial / \partial T$ is the transversal derivative with respect to $(\xi, \tau)$.

We also modify the definition of the Banach space $Z . Z$ is the set of all functions $u$ which are continuous in $\bar{D}_{\sigma}$ and which satisfy $u(x, t)=k(x, t)$ for $x \varepsilon \dot{B}_{2}$, $0 \leqq t \leqq \sigma$.

With the above modifications it is now easy to extend the proof of Theorem 3 to the present situation.

We can similarly extend Theorems $1^{\prime}, 2^{\prime}, 3^{\prime}, 3^{*}, \overline{3}$ to the present case.

We can also extend Theorem $4^{\prime}$. We have the following result: If

$$
\lim _{t \rightarrow \infty} \varphi(t)=A, \quad \lim _{t \rightarrow \infty} k(x, t)=A \quad \text { uniformly in } x \varepsilon \bar{B},
$$


then the solution $u(x, t)$ (which satisfies $\partial u / \partial T=g$ for $x \varepsilon \dot{B}_{1}, t>0$ and $u=k$ for $x \in \dot{B}_{2}, t>0$ ) satisfies

$$
\lim _{t \rightarrow \infty} u(x, t)=A \quad \text { uniformly in } x \varepsilon \bar{B} .
$$

5. Non-homogeneous Equations. In this chapter we generalize results obtained in previous chapters to the case of the non-homogeneous equation

$$
L u=f(x, t) .
$$

The uniqueness results (Theorems 1, 1') clearly hold for solutions of (5.1).

We proceed to generalize Theorem 2, assuming that $L$ satisfies (A), (B) and that $g(x, t, u)$ satisfies $\left(\mathrm{G}_{2}\right),\left(\mathrm{G}_{3}\right)$. We first introduce a function $\zeta(x)$ which satisfies (for some $\alpha>0$ )

$$
\begin{gathered}
\zeta(x) \geqq \alpha>0 \quad \text { on } \quad B, \\
L \zeta(x) \leqq-1 \quad \text { in } \quad D_{\tau}
\end{gathered}
$$

and let

$$
\left|\frac{\partial \zeta(x)}{\partial T}\right| \leqq \beta<\infty \quad \text { on } \quad S_{\tau} \text {. }
$$

We may, for instance, take $\zeta(x)=\gamma\left(e^{\delta R}-e^{\delta x_{1}}\right)$ where $\gamma$ and $\delta$ are appropriate constants and $R$ is such that $x_{1}<R$ for all $x=\left(x_{1}, \cdots, x_{n}\right)$ in $\bar{B}$.

Define

$$
K=\max \left({ }_{D} \text { l.u.b. }|f(x, t)|, \frac{1}{\alpha}{ }_{B} \text { l.u.b. }|\psi(x)|\right) .
$$

Then the function $w(x, t)=K \zeta(x)-u(x, t)$ satisfies

$$
\begin{gathered}
L w(x, t) \leqq 0 \text { in } D_{\tau}, \\
w(x, 0) \geqq 0 \text { on } \bar{B}, \\
\frac{\partial w}{\partial T}=K \frac{\partial \zeta}{\partial T}-g(x, t, K \zeta-w) \equiv \tilde{g}(x, t, w) \text { on } S_{\tau} .
\end{gathered}
$$

We shall assume that the following (physically obvious) condition is satisfied:

$$
\lim _{u \rightarrow \pm \infty} g(x, t, u)=\mp \infty \quad \text { uniformly in }(x, t) \varepsilon \bar{D}_{\tau} .
$$

It then follows from (5.7) that

$$
\tilde{g}(x, t, w) \geqq 0 \quad \text { if } \quad w<-H
$$

where $H$ is sufficiently large (depending on $g, K$ and $\beta$ ).

The method used to prove Theorem 2 can be applied to the present situation, on making use of (5.5), (5.6), (5.7) and (5.9). We then conclude that

$$
w(x, t)>-H \text { in } D_{\tau} \text {. }
$$


Using the definition of $w$ we get

$$
u(x, t) \leqq H+K \zeta(x) \leqq H^{\prime} \quad \text { in } \quad D_{\tau} .
$$

In a similar manner we obtain $u(x, t) \geqq-H^{\prime \prime}$ in $D_{r}$. Defining $H_{0}=$ $\max \left(H^{\prime}, H^{\prime \prime}\right)$, we have thus proved

Theorem $2^{\prime \prime}$. Let $L$ satisfy (A), (B) and let $g(x, t, u)$ satisfy $\left(\mathrm{G}_{2}\right),\left(\mathrm{G}_{3}\right)$ and (5.8). If $u(x, t)$ is a solution in $D_{\tau}$ of (5.1), (1.2), (1.3) and if it is continuous in $\bar{D}_{\tau}$, then

$$
|u(x, t)| \leqq H_{0} \quad \text { in } \quad D_{r}
$$

where $H_{0}$ depends only on $g$, the diameter of $B$, l.u.b. $|f(x, t)|$ in $D_{r}$, l.u.b. $|\psi(x)|$ in $B$, upper bounds on the coefficients $a_{11}(x, t),-b_{1}(x, t)$ of $L$ in $D_{r}$ and g.l.b. $a_{11}(x, t)$ in $D_{\tau}$.

Theorem $2^{\prime \prime}$ can easily be generalized to the case where the temperature of the gas $\varphi(t)$ varies in time.

With the aid of the uniqueness theorem and Theorem $2^{\prime \prime}$ we can now proceed to prove the existence of a solution in $D$ of the system (5.1), (1.2), (1.3) provided that, in addition to the assumptions made in the previous existence theorems, we assume also that $g$ satisfies (5.8) and that $f(x, t)$ is Hölder continuous in compact subsets of $\bar{D}$. The proof of the extension of Theorem 3 is similar to the proof of Theorem 3 with one modification: In defining the transformation $v=T u$ we add to the right side of (2.4) the generalized potential of volume distribution

$$
-\int_{0}^{t} \int_{B} 2 \rho(\xi, \tau) \Gamma(x, t ; \xi, \tau) f(\xi, \tau) d \xi d \tau
$$

whose properties have been studied in [12]. Theorems $3^{*}, \overline{3}, 3^{\prime}$ can also be generalized to the non-homogeneous equation (5.1). In what follows we shall refer to these extensions of Theorems $3,3^{*}, \overline{3}, 3^{\prime}$ as Theorem $3^{\prime \prime}$.

To prove an extension of Theorem $4^{\prime}$ we shall need the following assumptions:

$\left(\overline{\mathrm{G}}_{4}^{\prime \prime}\right)$ For any $K>0$ there exist positive constants $\eta, A$ such that for all $(x, t)$ in $\bar{D},|v| \leqq$ l.u.b. $|\varphi(t)|$ and $u^{\prime}>u^{\prime \prime},\left|u^{\prime}\right| \leqq K,\left|u^{\prime \prime}\right| \leqq K$

$$
-A\left(u^{\prime}-u^{\prime \prime}\right) \leqq g\left(x, t, u^{\prime}, v\right)-g\left(x, t, u^{\prime \prime}, v\right) \leqq-\eta\left(u^{\prime}-u^{\prime \prime}\right) .
$$

$\left(\overline{\mathrm{G}}_{6}\right)$ For every fixed $v$

$$
\lim _{u \rightarrow+\infty} g(x, t, u, v)=\mp \infty
$$

uniformly with respect to $(x, t)$ in compact subsets of $\bar{D}$.

Theorem $4^{\prime \prime}$. Let L satisfy (A), (B'), (C). Let $g(x, t, u, v)$ satisfy $\left(\overline{\mathrm{G}}_{1}\right),\left(\overline{\mathrm{G}}_{2}\right)$, $\left(\overline{\mathrm{G}}_{3}^{\prime}\right),\left(\overline{\mathrm{G}}_{4}^{\prime \prime}\right),\left(\overline{\mathrm{G}}_{6}\right)$. Let $f(x, t)$ be a bounded function in $\bar{D}$ which satisfies

$$
\lim _{t \rightarrow \infty} f(x, t)=0 \quad \text { uniformly in } x \in \bar{B}
$$


and assume that

$$
\varphi(\infty)=\lim _{t \rightarrow \infty} \varphi(t) \text { exists. }
$$

If $u(x, t)$ is a solution in $D$ of the system (5.1), (1.2), (4.1) and if it is continuous in $\bar{D}$, then

$$
\lim _{t \rightarrow \infty} u(x, t)=\varphi(\infty) \quad \text { uniformly in } x \varepsilon \bar{B}
$$

Proof. Let $\epsilon$ be an arbitrary positive number and let $\sigma$ be such that

$$
\begin{gathered}
|f(x, t)|<\epsilon \quad \text { if } t \geqq \sigma, \quad x \varepsilon \bar{B}, \\
|\varphi(t)-\varphi(\infty)|<\epsilon \text { if } t \geqq \sigma .
\end{gathered}
$$

Let $|u(x ; \sigma)| \leqq C_{1}$ for $x \varepsilon \bar{B}$ and denote by $v$ the solution of the system

$$
\begin{gathered}
L v=-\epsilon \text { in } D-\bar{D}_{\sigma}, \\
v(x, 0)=C_{1} \text { for } x \varepsilon \bar{B}, \\
\frac{\partial v}{\partial T}=g\left(x, t, v, \varphi(\infty)+\epsilon_{+}\right) \text {on } S-\bar{S}_{\sigma},
\end{gathered}
$$

where $\epsilon_{+}=\min (\epsilon$, l.u.b. $\varphi(t)-\varphi(\infty))$. The existence of $v$ follows from Theorem $3^{\prime \prime}$. By a simple comparison argument we derive the inequality

$$
u(x, t) \leqq v(x, t) \text { in } D-D_{\sigma},
$$

and we have to estimate $v$. To do that define

$$
w=v+\epsilon \vartheta(x)
$$

where $\vartheta(x)$ is any function which satisfies $L \vartheta \geqq 1$.

Because of (5.15) $w$ satisfies the system

$$
\begin{gathered}
L w \geqq 0 \quad \text { in } D-\bar{D}_{\sigma}, \\
w(x, \sigma) \leqq C_{2} \text { for } x \varepsilon \bar{B} \quad\left(C_{2}=C_{1}+\epsilon \text { l.u.b. }|\vartheta(x)|\right), \\
\frac{\partial w}{\partial T}=g\left(x, t, w-\epsilon \vartheta, \varphi(\infty)+\epsilon_{+}\right)+\epsilon \frac{\partial \vartheta}{\partial T} \equiv g_{0}(x, t, w) \quad \text { on } \quad S-\bar{S}_{\sigma} .
\end{gathered}
$$

Using $\left(\overline{\mathrm{G}}_{4}^{\prime \prime}\right)$ it follows that there exists a sufficiently large constant $N$ such that

$$
g_{0}(x, t, w) \leqq g\left(x, t, w-N \epsilon, \varphi(\infty)+\epsilon_{+}\right) .
$$

We now denote by $s(x, t)$ the solution of the system

$$
\begin{gathered}
L s=0 \text { in } D-\bar{D}_{\sigma}, \\
s(x, \sigma)=C_{2} \text { for } x \varepsilon \bar{B}, \\
\frac{\partial s}{\partial T}=g\left(x, t, s-N \epsilon, \varphi(\infty)+\epsilon_{+}\right) .
\end{gathered}
$$

The existence of $s$ follows from Theorem $\overline{3}$. 
Using (5.19) and applying Lemma $2^{\prime}$ we get

$$
w(x, t) \leqq s(x, t) \quad \text { in } \quad D-D_{\sigma} .
$$

Applying Theorem 4 to the solution $s(x, t)$ of the system (5.20) we get

$$
s(x, t)-\left(\varphi(\infty)+\epsilon_{+}+N \epsilon\right)=O(\exp \{-\mu t\}) \quad(\mu>0)
$$

provided $t \geqq t^{\prime}$ and $t^{\prime}$ is sufficiently large.

Combining (5.22) with (5.21), (5.17) and (5.16) we get

$$
u(x, t) \leqq \varphi(\infty)+C_{3} \epsilon \quad\left(C_{3}>0\right)
$$

provided $t \geqq t_{1}$ and $t_{1}$ is sufficiently large $\left(C_{3}\right.$ is independent of $\epsilon$ and $\left.t_{1}\right)$. In a similar way we get $u(x, t) \geqq \varphi(\infty)-C_{4} \epsilon$, and the proof of Theorem $4^{\prime \prime}$ is thereby completed.

We remark that the proof of Theorem $4^{\prime \prime}$ remains valid under weaker assumptions than $\left(\overline{\mathrm{G}}_{4}^{\prime \prime}\right)$ and $\left(\overline{\mathrm{G}}_{6}\right)$. Thus it is enough to assume that the inequalities in $\left(\overline{\mathrm{G}}_{4}^{\prime \prime}\right)$ hold only for an appropriate large $K$ and, instead of assuming $\left(\overline{\mathrm{G}}_{6}\right)$, to assume only that $g$ becomes sufficiently large (small) for some $u>0(u<0)$.

Theorem $4^{\prime \prime}$ was proved under the assumption $c(x, t) \equiv 0$. We can now generalize Theorem $4^{\prime \prime}$ by replacing this assumption by the weaker assumption

$$
\lim _{t \rightarrow \infty} c(x, t)=0 \quad \text { uniformly in } x \varepsilon \bar{B} .
$$

Indeed, we have just to transfer $c(x, t) u(x, t)$ from the left side of (5.1) to the right side, note (on using Theorem $2^{\prime \prime}$ ) that $\lim _{t \rightarrow \infty} c(x, t) u(x, t)=0$ and apply Theorem $4^{\prime \prime}$.

We can similarly extend Theorems $4,4^{\prime}$ to the case that $c(x, t)$ satisfies (5.24). We remark that if $\lim _{t \rightarrow \infty} c(x, t) \neq 0$ and if $\varphi(\infty) \neq 0$ then $v(x) \equiv \varphi(\infty)$ is not a solution of the system $\left(\lim _{t \rightarrow \infty} L\right) v=0$ in $B, \partial v / \partial T=0$ on $\dot{B}$ and therefore we should not expect that $\lim _{t \rightarrow \infty} u(x, t)=\varphi(\infty)$.

Theorem $5^{\prime}$ can also be extended to the case of the non-homogeneous equation (5.1), provided $f$ satisfies

$$
f(x, t) \leqq 0, \quad f(x, t+h) \leqq f(x, t)
$$

for all $(x, t) \varepsilon \bar{D}$ and $h>0$.

\section{References}

[1] F. G. Dressen, The fundamental solution of the parabolic equation, Duke Math. J., 7 (1940), pp. 186-203.

[2] F. G. Dressen, The fundamental solution of the parabolic equation II, Duke Math. $J ., 13$ (1946), pp. 61-70.

[3] A. Friedman, Remarks on the maximum principle for parabolic equations and its applications, Pacific J. Math., 8 (1958), pp. 201-211.

[4] A. Friedman, Parabolic equations of the second order, to appear in Trans. Amer. Math. Soc.

[5] A. Friedman, Heat transfer between solids and gases under nonlinear boundary conditions, Technical Report, August 1958, University of California, Berkeley, California. 
[6] M. Gevrex, Sur les equations aux derivees partielles du type parabolique, J. Math. Pures et Appl., 9 (ser. 6) (1913), pp. 305-471.

[7] W. R. MANN \& F. WoLF, Heat transfer between solids and gases under nonlinear boundary conditions, Quarterly Appl. Math., 9 (1951), pp. 163-184.

[8] L. Nirenberg, A strong maximum principle for parabolic equations, Comm. Pure Appl. Math. 6 (1953), pp. 167-177.

[9] K. Padmavally, On a non-linear integral equation, J. Math. and Mech., 7 (1958), pp. 533-555.

[10] W. Pogonzelski, Sur le problème de Fourier généralisé, Annal. Polon. Math., 3 (1956), pp. 126-141.

[11] W. Pogonzelski, Étude de la solution fondamentale de l'équation parabolique, Ricerche di Mat., 5 (1956), pp. 25-57.

[12] W. Pogonzelski, Propriétés des integrales de l'équation parabolique normal, Annal. Polon. Math., 4 (1957), pp. 61-92.

[13] W. Pogonzelski, Problèmes aux limites pour l'équation parabolique normale, Annal. Polon. Math., 4 (1957), pp. 110-126.

[14] W. PagorzeLski, Problème aux limites pour l'équation parabolique dont les coëfficients dépendent de la fonction inconnue, Ricerche di Mat., 5 (1956), pp. 258-271.

[15] J. H. Roberts \& W. R. ManN, A nonlinear integral equation of Volterra type, Pacific J. Math., 1 (1951), pp. 431-445.

[16] J. SchaUdER, Der Fixpunktsatz in Funktionalraümen, Studia Math., 2 (1930), pp. 171-180.

University of California

Berkeley, California

and

Indiana University

Bloomington, Indiana 